\title{
ADaPPT: Enterprise Architecture Thinking for Information Systems Development
}

\author{
Hanifa Shah and Paul Golder \\ Birmingham City University, \\ TEE, Millennium Point, Birmingham, B4 7XG, UK
}

\begin{abstract}
Enterprises have architecture: whether it is visible or invisible is another matter. An enterprises' architecture determines the way in which it works to deliver its business objectives and the way in which it can change to continue to meet its evolving business objectives. Enterprise architectural thinking can facilitate effective strategic planning and information systems development. This paper reviews enterprise architecture (EA) and its concepts. It briefly considers EA frameworks. It describes the ADaPPT (Aligning Data, People, Processes and Technology) EA approach as a means to managing organisational complexity and change. Future research directions are discussed.
\end{abstract}

Keywords: Business Strategies, Enterprise Architecture, Organisational Processes, Enterprise Planning, Technologies Alignment.

\section{Introduction}

Much progress has been made in recent years in developing structures to describe the enterprise and to facilitate the development of information systems that appropriately complement the strategy of the enterprise. Despite the success of the enterprise architecture approach there are still major problems in achieving organisational change and in driving the re-alignment of IT systems. The complexity of modern organisations in terms of the business, legal and technological environment demands an architectural approach. Businesses are faced with ongoing and continual change to which they must respond in order to ensure success and even survival. The increasingly competitive environment demands a customer-focused approach. All of these factors contribute to the complexity and uncertainty faced by organisations resulting in an inability to be appropriately responsive to both internal and external events. Underlying this complexity and uncertainty is the gap between an organisation's business objectives and its underlying IT infrastructure. There is a need for information that is timely and understood in order to facilitate appropriate analysis and to appreciate the relevant impacts of decisions made. Organisations are continually faced with the challenge of their IT delivering the business value demanded and responding speedily to the changing business needs.

\section{Enterprise Architectural Thinking}

An EA approach can help to provide a vehicle for organisational communication. Improving communication and discussion between business and IT staff enabling a shared understanding of the business and its supporting infrastructure that can facilitate improved decision making and more effective deployment of change. The approach provides a basis for standardisation and agreed notations and representations, processes and information become more transparent. Project costs can become more stable and better predicted, the time taken to bring about change either by enhancing current services or by offering new ones can be reduced.

Clearly identifying the key components through an enterprise architecture approach of business processes, information, technology applications and organisation and how these relate to each other facilitates focussing on the appropriate component as required in a particular situation. EA can be used to manage complexity and describe the interdependencies in a usable manner.

EA can facilitate a better return on an organisations investment by providing a means to identify cost saving opportunities, gaps and inconsistencies as well as facilitating the installed systems and applications being exploited. An enterprise architecture approach leads to improved scoping and coordination of programmes and projects. 


\section{The Challenge of Change}

It is commonplace to identify the forces of change to which modern businesses are exposed. It is relevant to discriminate between forces for change that affect the business being carried out and those which affect only the way the business is delivered. A new computer system leaves organisational models unchanged but may change the data models and applications used to support them. A change in market or a merger will change the organisational model itself. The pressures for change on the organisation are such that a process of continual evolution even revolution is affecting all organisations. This means that the construction of an enterprise architecture is not a single event generating a static description of a the organisation which thereafter impedes the process of change. On the contrary the continual evolution of the enterprise architecture is a process in parallel with the evolution of the business strategy. The question should be asked how do we architect the business to meet its evolving strategic needs and the answer should lie in the continual evolution of the architecture. The architecture is the interface between the strategic, what the enterprise wants to do, and the operational, what it does.

Strategic change in the organisation can lead to evolutionary changes in the enterprise architecture but may require more radical change. For example the merging of two organisations may require the integration of their existing enterprise architectures into a new common EA. This is a similar problem to the evolving enterprise architecture one but is likely to require more substantive change. For example we may not be able to assume that the concept 'Customer' is exactly the same in the two merging organisations so may need to examine this at some detail in order to achieve successful integration. However in an organisation that is evolving from concrete to virtual trading, the concept of customer may be undergoing equally significant change and the significance of this change may be overlooked in the assumption that it is evolutionary.

An organisation has a business strategy at a particular time; corresponding to this strategy it has (or is in the process of developing) the corresponding enterprise architecture for delivering this strategy. That existing enterprise architecture describes and specifies a number of business processes, data objects and applications which 'operationalises' the architecture. Next the business introduces a new strategy, corresponding to this we have desired enterprise architecture and its corresponding business processes, data objects and applications. The practical problem becomes how do we migrate from one EA to the next? Moreover we would want to know the series of architectures (or roadmap) that would take us through the required transitional architectures. How do we identify the changes necessary in business processes, data objects, and applications required and how do we manage the transitions.

The following examples serve to highlight what is needed in the management of organisational change through evolving enterprise architectures.

- We need to be able to examine the ontology of concepts - what is a customer?

- We need to be able to identify the dynamics of the elements - how does a customer come into existence, what determines the life of a customer, how is it terminated?

- We need to be able to identify the agents responsible who authorises the creation of a customer, who determines when a customer is no longer?

- We need to be able to specify the business rules related to the behaviour of customers and agents.

Existing methodologies and tools do not help use with these problems they are mainly focused on the storage and retrieval of data, and the specification of data manipulation processes. An enterprise architectural approach can facilitate this thinking.

Enterprise architecture has been widely adopted as a means to cope with the ever-increasing complexity of organizations and to ensure that the technical resources are appropriately employed and optimized [1]. EA is the fundamental organization of the system, embodied in its elements, their relationships to each other and to the environment and the principles guiding its design and evolution [2], [3]. Enterprise architecture is described as organizing logic for business processes and IT infrastructure, reflecting the integration and standardization requirements of the company's operating model in order to achieve business agility and profitable growth [4]. Currently, there exist a number of professional societies and organizations that are working on the definition and the management of enterprise architecture such as The Open Group, Microsoft, and IBM. Indeed, EA represents much more than IT architecture. It is an integrated and holistic vision of how the business processes across the enterprise, people, information, applications and technologies align to facilitate strategic objectives. EA frameworks identify the scope of EA and decompose various elements of the architecture onto structured layers/levels and elements. Several EA frameworks have been adopted for operational use in many private and governmental organizations. 
EA emerged as an idea in 1980 and is embodied in the early EA framework developed by Zachman (1987) [5]. EA has re-emerged as a means to cope with the everincreasing complexity of organizations. This re-emergence is closely related to the evolution of new business trends and to the evolution of IT, particularly to the advances in Internet technologies. These business trends comprise globalization, mergers and acquisitions, e-commerce, as well as the increasing importance of customer relationship management (CRM) and supply chain management. IT trends, on the other hand, comprise the advances in Internet technologies, hardware platform, application servers, and workflow servers. Due to the increasing importance of EA, certification opportunities in EA are being offered by several companies such as The Open Group and IBM in order to standardize an open method for IT architecture to solve business problems.

An EA approach is beneficial in aligning business and IT resources and in conforming to fundamental principles and common methodologies that govern the entire life cycle of the IS development process. In that sense, architectural frameworks are considered to be a convenient way to support such methodologies, and to separate roles that facilitate and implement these methodologies as needed. Still, there are many organizational and technical EA challenges.

\section{Enterprise Architecture Framework}

EA frameworks identify the scope of the enterprise architecture and decompose various elements of the architecture onto structured levels and elements [6]. More formally, EA frameworks describe a method for designing IS in terms of a set of building blocks and how these blocks fit together. Several EA frameworks such as ARIS [7] and DODAF [3] have been adopted for operational use in many organizations. For example, the Federal EA [8], has been adopted by the US government as a businessdriven framework in order to optimize some strategic areas. These areas include budget allocation, information sharing, performance measurement, and component based architecture. More specifically, EA frameworks contain a list of recommended standards and compliant products that are used to implement the building blocks for an IS. EA frameworks are useful in terms of simplifying architecture development and ensuring complete coverage of the designed solutions through a common terminology. In that sense, these frameworks are language independent by providing generic concepts and common terminology through which different EA stakeholders can communicate without making any assumptions about each others' language. Pragmatically, EA frameworks play a dual role. Firstly, they serve as implementation tools; secondly, they can serve as organisational planning tools.

\section{The ADaPPT Approach}

Fig. 1 ADaPPT Approach.

ADaPPT was developed in work with organisations using the ALTAR (Achieving Learning Through Action Research) methodology [1]. ADaPPT has four domains (elements ${ }^{1}$ ): people, processes, technology and data.
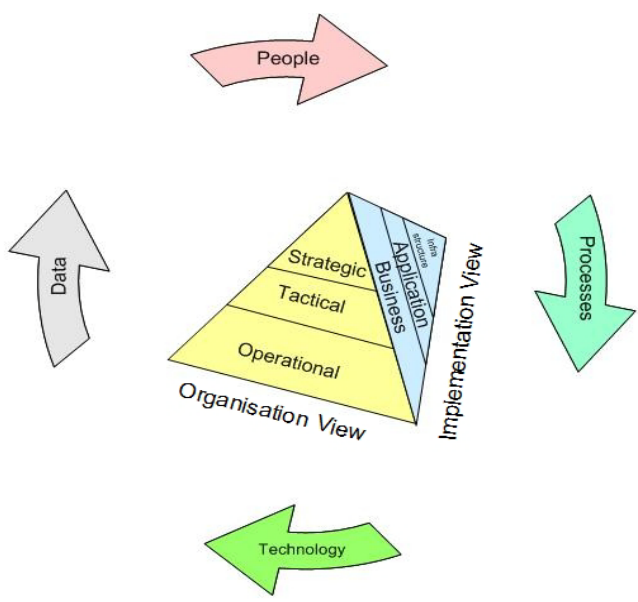

ADaPPT : Aligning Data, People, Processes and Technology There are two main views in ADaPPT: a organisational view and an implementation view. The model recognises that everything the enterprise does involves people, processes, technology and data and that these need to be aligned.

\section{'A process driven by people consumes resources (technology) and generates data'}

In the ADaPPT framework the term people represents not only individual people but groups within the organisation, departments, sections etc. and also roles such as marketing manager etc. In as much as they can initiate actions and be responsible for the processes of the organisation. The term agent is also used.

In ADaPPT process means all activity and actions within the organisation. This includes high level business processes - marketing, production etc.; middle level activities - launching new products etc; operational activities - checking an invoice - etc.

\footnotetext{
${ }^{1}$ We call these elements because just as the ancients believed that every thing was composed of the four basic elements, Fire, Water, Air, Earth. So ADaPPT believes that every business activity combines the basic elements of, People, Process, Data, Technology.
} 
In ADaPPT technology includes all services, material and equipment used by the organisation. This includes computer and IT hardware and software, raw materials and processed product.

In ADaPPT data means all information both static and dynamic within the organisation such as management targets, performance measures and operational data : customer details etc.

The organisational view in ADaPPT recognises that the nature the enterprise varies throughout the organisational hierarchy:

\section{- $\quad$ Strategic \\ - Tactical \\ - Operational}

As we climb the hierarchy processes become less well defined, soft data becomes more important, the scope of responsibility becomes wider. ADaPPT does not attempt to be a complete framework for all enterprise needs it is focused on the business / technology issues.

The implementation view recognises that there is a spectrum from business through application to infrastructure. This can apply equally to each domain. For example data can be viewed at: the business level expressed as E-R and other data models; at the application level expressed as data structure diagrams; at the infrastructure level expressed through allocation of data on storage devices. If we consider the interactions between views we will see that the implementation view and the business views are independent of each other. Thus an operational level business problem will need to be addressed at the business level as a specification of the business problem, at the application level as design of the solution to the problem and at the infrastructure level by provision of software and other resources to implement the solution.

The ADaPPT framework thus has four domains each of which can be described with a three by three matrix of views.

'This is because enterprise planning is a complex process involving many thousands of elements. The ADaPPT approach aims to organise and simplify the process of thinking about and managing these thousands of elements'.

\subsection{ADaPPT as Implementation Tool}

ADaPPT in common with other EA frameworks provides a comprehensive representation of IS in terms of its building blocks. In this context, ADaPPT relates the necessary IS aspects/dimensions such as business processes, data, and organization units to different perspectives at certain levels of abstraction. These perspectives rely mainly on the difference in EA stakeholders' views of the architecture that span different level of details. EA frameworks, as components specification tools, encompass the documentation of the architectural layers, architectural domains, architectural models, and architectural artefacts.

Typically, EA frameworks such as ADaPPT are decomposed to three architectural layers, which are business layer, application layer, and technology infrastructure layer [9]. The business layer describes the business entities such as business processes and relevant business information, and how these entities interact with each other to achieve enterprise wide objectives. The application layer determines the data elements and the software applications that support the business layer. The technology infrastructure layer comprises the hardware platforms and the communication infrastructure that supports the applications. Such layers are naturally characterized by information aspects, behavioural aspects, and structural aspects. As organizations consist of several units, the structural aspects determine the static decomposition of these units to several sub-units. The behavioural aspects show behaviour manifested in the sequence of activities and business processes performed to produce the needed services. These units exchange information in order to carry out business tasks. Each layer is naturally composed of several domains that reflect the information, behavioural, and structural aspects of the organizations. These domains specify the architectural aspects such as process architecture, product architecture, information architecture, technical architecture, and application architecture. Indeed, these domains are the means to separate the architectural concerns and reflect the view of different EA stakeholders to the architecture. For example, the process domain, which is a part of the business layer, describes business processes or business functions that offer the products or services to an organization. These architectural domains are typically described and documented by different architectural models such as business process models, value chain diagrams, and organization charts. Architectural models serve as a basis for documenting the different architectures by annotating the artefacts and their inter-relationship that are needed to model an organization from different perspectives. Architectural artefacts represent the necessary constructs and architectural elements such as data, business processes, resources, and events that represent the real world objects needed to design distinct model types. 


\subsection{ADaPPT as Implementation Tool}

ADaPPT in common with other some other EA frameworks provide a holistic view of EA through the hierarchical layering, which implies the alignment between, business, application, and technology infrastructure layer. As such, business decisions and architecture planning can be made in the context of whole instead of standalone parts. In other words, EA frameworks such as ADaPPT make use of the abstractions in order to simplify and isolate simple IS aspects/dimensions without losing sense of the complexity of the enterprise as a whole. As an organisation planning tools, ADaPPT entail baseline architecture, future architecture, architectural roadmaps, and transition plans. Baseline architecture, which is also known as 'as-is' view, encompasses the documentation of different layers and the existing components (models, diagrams, documents etc). This architecture serves as a baseline for identifying the relationships between different components and the gaps that should be filled for better organizational performance. Target architecture, which is also referred to as the 'to be' view, specify the new EA components and the strategic initiatives that should be carried out for the sake of bridging the gaps and ensuring competitive advantage. This architecture should also identify the IT resources and technological infrastructure that are needed for supporting the new EA components in order to integrate the organization structure, business processes, data, and technical resources. Architectural roadmaps represent the intermediary EA alternatives of the baseline architecture generated in the process mitigating the risks and analyzing the existing gaps in order to shift to the target architecture. These roadmaps annotate the architectural milestones performed prior to reaching the target architecture. EA transition plans are merely specifications of an 'as-is' and 'to-be' view in terms of managing the feasibility of architectural transition such as risk assessment, gap analysis, and the supporting resources of the transition. More specifically, transition plans document the activities that need to be undertaken to shift from the baseline architecture to the target architecture. Such plans are means to determine the desired future state of the enterprise wide goals, business processes, technical resources, organization units, and data.

\subsection{ADaPPT and other EA Frameworks and Tools}

A range of tools can be used to model the architecture appropriate to the different views. Where appropriate familiar tools are used across several views so we do not need 36 different models as in the Zachman [5] approach eg E-R modelling is used for the Data Domain and UML can be used in the Process Domain Business and
Application Views. Figure 2 illustrates a mapping between the Zachman approach and ADaPPT.

\begin{tabular}{|l|c|c|c|c|c|c|}
\hline \multicolumn{3}{|c|}{ Zachman } & & & \\
\hline AdaPPT & & & & & & \\
Domains & Data & Function & Network & People & Time & Motivation \\
\hline People & & & X & X & X & X \\
\hline Process & & X & X & & X & \\
\hline Technology & & & X & & X & \\
\hline Data & X & & X & & X & \\
\hline
\end{tabular}

Fig. 2 ADaPPT and Zachman Framework.

One of the leading EA toolset is ARIS [7]. Whilst a toolset may support many frameworks it will also have an implicit ontology. ARIS is a complex tool with an underlying Process Model. ARIS manages complexity with four Views: Data View, Organization View, Function view, Product Service View. ARIS supports a detailed process oriented view of the organisation. However the basic units of ARIS fit easily within the ADaPPT framework. There is no inconsistency in using ADaPPT as an EA Framework and ARIS as the toolset to support the management and operation or the Enterprise's Architecture Repository. The four ARIS views are apparently consistent with the ADaPPT framework. However it is worth examining some of the lower level ARIS concepts to see if this apparent alignment in high level concepts is reflected in the detail (see Figure 3).
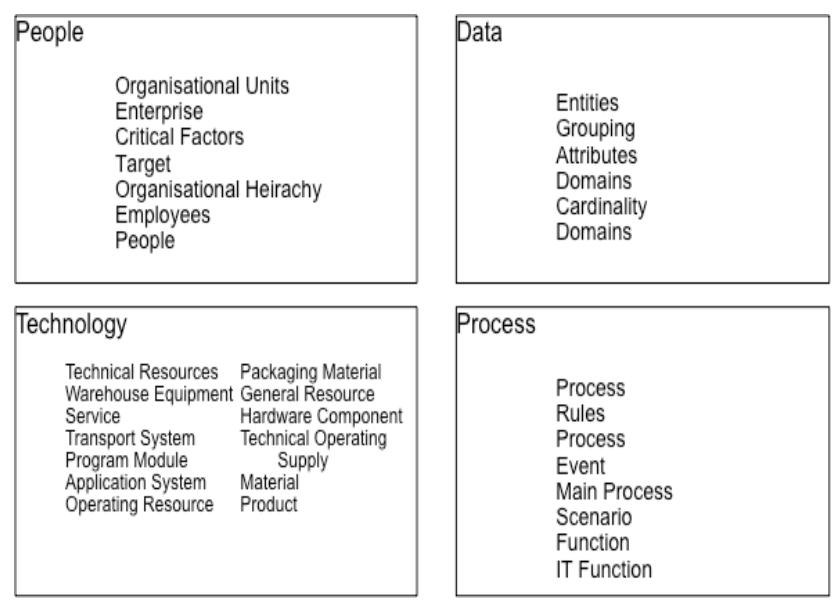

Fig. 3 ARIS concepts within ADaPPT Domain.

In ADaPPT it is possible to use conventional diagramming tools such as MS Visio or complex EA diagrammers or even full blown modellers such as IDS Sheer. It is the way the different elements combine which creates business value.

The main relationships in ADaPPT (see figure 4) are: 
People: Initiate processes, Use data, Specify technology

Processes: Run on technology, Use technology, Generate data

Data: Stored on technology

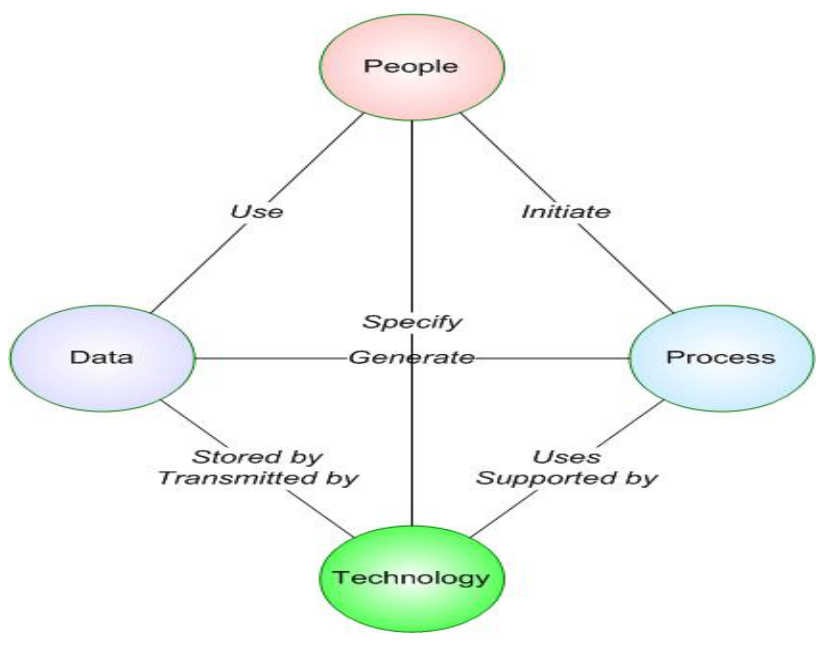

Fig. 4 The main relationships in ADaPPT.

These can be represented in the various EA tools. These main relationships are important. Representing, exploring and planning related to these elements is facilitated by, recognising and taking account of the main relationships and their content from organisational and implementation perspectives as appropriate for the particular context under consideration.

\section{Conclusions}

Our ongoing work is applying ADaPPT based enterprise architecture thinking for information systems development in public and private sector organisations. It provides a strong foundation for understanding the strategic, managerial and operational issues in aligning people, processes, data and technology and also in developing strategic, managerial and operational approaches while ensuring the alignment of people, processes, data and technology in an IS context. It is being used as the basis for understanding how knowledge management can be improved and new technologies exploited by organisations. Providing a basis for enabling the conceptualising of holistic, integrated and detailed consideration as appropriate to the development stage and stakeholder perspective.

\section{References}

[1] Author, Eardley WA and Wood-Harper AT (2007) ALTAR: Achieving Learning Through Action Research, European Journal of Information Systems, Vol 16 No 6, Pp. 761-770.

[2] IEEE (2006), "IEEE Standards Association, IEEE Std 14712000 IEEE Recommended Practice for Architectural Description of Software-Intensive Systems, http://standards.ieee.org/reading/ieee/std_public/description/s e/1471-2000_desc.html," 2006.

[3] DOD (2006), "DOD Architecture Framework, Systems and Software Consortium, http://www.software.org/pub /architecture/dodaf.asp," 2006.

[4] J. W. Ross, P. Weill, and D. C. Robertson (2006), Enterprise Architecture as Strategy: Creating a Foundation for Business Execution. Boston, Massachausettes: Harvard Business School Press Book.

[5] J. A. Zachman (1987) "A Framework for Information Systems Architecture," IBM Systems Journal, Vol. 26, No. 3.

[6] Author and El Kourdi M (2007), Enterprise Architecture Frameworks, IEEE IT Professional, Vol 9, No 5.

[7] A.-W. Scheer (1999), Business Process Engineering: Reference Models for Industrial Enterprises, 2nd ed. berlin: Springer.

[8] FEA (2003) CIO, " Practical Guide to Federal Enterprise Architecture," Enterprise Architecture Program Management Office, http://www.feapmo.gov.

[9] H. Jonkers R. v. Buuren, F. Arbab, F. d. Boer, M. Bonsangue, H. Bosma, H. t. Doest, L. Groenewegen, J. G. Scholten, S. J. B. A. Hoppenbrouwers, M. E. Iacob, W. Janssen, M. M. Lankhorst, D. v. Leeuwen, H. A. Proper, A. Stam, L. v. d. Torre, and G. E. V. v. Zanten, (2003), "Towards a Language for Coherent Enterprise Architecture Descriptions," in 7th IEEE International Enterprise Distributed Object Computing Conference (EDOC 2003). Brisbane, Australia, 2003.

Hanifa Shah is Associate Dean (Research) and Professor of Information Systems at Birmingham City University. She provides leadership and strategic direction to the research of the Faculty of Technology, Engineering and the Environment where staff are involved in research in three Centres. In the Centre for Low Carbon Research (CLCR) staff are investigating bio-energy generation, low carbon transportation, sustainable, intelligent buildings and retrofit and knowledge based engineering. In the Institute for Digital Experience and Applications (IDEAS) staff are contributing to research to help maximise economic and societal benefits of digital technologies. The Centre for Environment and Society Research (CESR) considers how planning policy and technology affect trends both in society and the environment in light of historical, contemporary and future contexts. Prof Shah was previously at Staffordshire University and at Aston University and is currently Visiting Professor at Manchester University. She has over 30 years in-depth knowledge and experience of both the higher education sector and business. Prof Shah has been at the forefront of the development of industrial links and partnerships for research, enterprise and teaching by Universities. She has supervised over twenty PhDs and has specialized in linking PhDs to industrial collaborations producing results with practical impact as well as academic significance. She has been invited to advise public and private sector organizations on strategic IT issues and also on the professional development of IT staff and universityacademic collaboration opportunities. Prof Shah has helped to shape national and international thinking on work-based learning and university qualifications for IT Professionals through her work 
on transformational development programmes for large corporations and public sector organizations. As a result of her work she has published over seventy papers, a number of book chapters and a book. Prof Shah's current research includes research methodologies for information systems research with collaborating corporations (ALTAR), organisational and professional development in IT (STEP) and the exploitation of mobile technologies in health informatics and the improvement of patient processes in hospitals. She is investigating enterprise architecture based approaches for information systems and knowledge management (ADaPPT) in areas such as healthcare and e-government.

Dr Paul Golder is a Visiting Research Fellow in the Faculty of Technology, Engineering and the Environment at Birmingham City University. His career has included time as an applied statistician with periods in various public sector organisations including the European Commission in Luxembourg. After that he concentrated on the data management aspects of decision making within the Computer Science Group of Aston University, and at the same time collaborated with colleagues within Aston Business School (ABS). Since retiring from Aston University in 2004 he has remained active in research continuing to collaborate with colleagues at Staffordshire University where he was appointed Visiting Research Fellow, during this time he undertook a part time employment as a Research Fellow to bring to completion an EPSRC funded research project. He has continued to assist in the supervision of PhD students and to carry out industrial placement visits for $A B S$. He has substantial experience in all areas of information management. $\mathrm{He}$ has expertise both in the development of information strategies and also skills in many of the areas central to the successful implementation of an information strategy. biography appears here. Degrees achieved followed by current employment are listed, plus any major academic achievements. Do not specify email address here. 